\title{
Spectral switching control of ultrafast pulses in dual core photonic crystal fibre
}

\section{Koys}

\section{Bugar \\ bugar@ilc.sk}

\section{Hrebikova}

\section{Mesaros}

\section{R. Buczynski}

\section{F. Uherek}

International Laser Centre, Ilkovicova 3, 84104 Bratislava, Slovakia

International Laser Centre, Ilkovicova 3, 84104 Bratislava, Slovakia

Faculty of Mathematics, Physics and Informatics, Comenius University, Mlynska Dolina, 84248 , Bratislava, Slovakia

Faculty of Mathematics, Physics and Informatics, Comenius University, Mlynska Dolina, 842 48, Bratislava, Slovakia

Faculty of Electrical Engineering, Czech Technical University in Prague, Technicka 2, 16627 Prague, Czech Republic

Faculty of Mathematics, Physics and Informatics, Comenius University, Mlynska Dolina, 84248 , Bratislava, Slovakia

Institute of Electronic Materials Technology, Wolczynska 133, 01-919 Warsaw, Poland

The work presented in this paper is a study of an all-optical narrow-band switch in extended spectral area by dual core photonic crystal fibre expressing nonlinear coupler performance. The investigation is focused on the nonlinear propagation of femtosecond pulses in the near infrared spectral region at up to $50 \mathrm{~kW}$ peak power which induces spectral broadening through almost two octaves. The mutual effect of nonlinear spectral transformation and field redistribution between the two fibre cores is analyzed by both theoretical and experimental approaches. The simulation of the nonlinear propagation is based on coupled generalized nonlinear Schrödinger equations. A modified numerical model utilizing split-step Fourier method was adapted for dual core fibres. The complex experimental study was accomplished for various input settings such as polarization, intensity and selective coupling into each core and the selective detection of spectra from each core. The presented work encompasses promising results obtained regarding a spectral intensity switch between the two output channels by input intensity or polarization change in the S-band of optical communication systems.

[DOI: http://dx.doi.org/10.2971/jeos.2013.13041]

Keywords: Dual core fibre, photonic crystal fibre, nonlinear propagation, all-optical switch, coupled generalized nonlinear Schrödinger equations

\section{INTRODUCTION}

Photonic crystal fibres (PCF) have extraordinary properties comparing to standard optical fibres. PCF technology offers great possibilities in tailoring the guiding properties of the fibre by modifying the cladding structure design [1, 2]. Some examples of special PCFs are endlessly single-mode, large mode area, double cladding or dual core structures. All of the mentioned special types of fibres are hard or impossible to manufacture by the technologies utilized for the production of standard optical fibres. The various types of PCF offer outstanding solutions for different applications such as dispersion control [3], supercontinuum generation [4], nonlinear coupling [5] and sensing tasks. In this paper we focus on the nonlinear all-optical spectral intensity switch of spectrally broadened femtosecond pulses in a dual core PCF.

PCF technology has led to the revitalization of nonlinear optics since the use of fibre optics generally ensures a long interaction length and, moreover, the PCF technology enhances the localization of the light in an extremely small core. The second key advantage of light guiding by PCF is the possibility of boosting the nonlinear effects by adjusting the fibre dispersion. One of the most intensively studied phenomena that exploits nonlinear propagation in PCFs is that of supercontinuum generation. This effect occurs when a spectrally narrow input pulse undergoes significant spectral broadening in the PCF fibre resulting in a broad spectrum (of one or more octaves width) at the output. The most important nonlinear processes governing the supercontinuum generation are self-phase modulation (SPM), cross-phase modulation (XPM), spontaneous and stimulated Raman scattering processes and, in the anomalous dispersion region, modulation instability, solitary wave generation and dispersion wave generation. The evaluation of the impact of the individual nonlinear effects from the large ensemble of potential processes is the key task for a proper description of the nonlinear propagation of light in the PCF. A detailed analysis of the effects influencing the 
supercontinuum generation is contained in the review paper of Dudley [6].

The key phenomenon in the case of dual core fibre propagation is the coupling, which means light energy transfer from one core to the other after a characteristic distance called the coupling length. Coupling can be explained in terms of intermodal interference of supermodes, which are the eigenmodes of the whole dual core structure. There are two fundamental supermodes - symmetric and anti-symmetric. The symmetric supermode field has the same phase and the antisymmetric has the opposite phase comparing the two cores of symmetric dual core structure. A different theoretical approach to describe the coupling between the cores is based on the modal overlap of the individual modes propagating in each core (coupled mode theory). Both interpretations are equivalent as the properties of the fibre can be recalculated in terms of the other interpretation by expressing the total electric field as the sum of the electric fields in the individual cores [7]

$$
E(x, y, z, \omega) \approx E_{1}(x, y, z, \omega)+E_{2}(x, y, z, \omega)
$$

where $E(x, y, z, \omega)$ is the electric field in whole structure, while $E_{1}(x, y, z, \omega)$ and $E_{2}(x, y, z, \omega)$ are the electric fields in the individual cores and $\omega$ is the light carrier frequency. The coupling length $L_{\mathcal{C}}$ is defined for both interpretations as [7]

$$
L_{c}=\frac{\pi}{\left|\beta_{S}-\beta_{A}\right|}=\frac{\pi}{\sqrt{\left(\beta^{(1)}-\beta^{(2)}\right)^{2}+4 \kappa^{(1)} \mathcal{K}^{(2)}}}
$$

where $\beta_{S}$ and $\beta_{A}$ are the propagation constants of the symmetric and anti-symmetric supermodes respectively. In terms of coupled mode theory, $\beta^{(1)}$ and $\beta^{(2)}$ are the propagation constants of each separate core and $\kappa^{(1)}$ and $\kappa^{(2)}$ are the coupling coefficients characterizing the field overlap of the second core with the first and the first core with the second respectively.

The shift towards the new technology of PCF production has resulted in easier dual core fibre preparation compared to standard fibres. In the case of PCFs there are much wider possibilities to shape the coupling properties compared to standard dual core fibres. The dual core photonic crystal fibres (DC PCF) operating in the nonlinear regime are commonly investigated as all-optical nonlinear fibre couplers. The majority of the previous papers covering these fibres $[8,9]$ have presented mainly the theoretical results of the coupling properties of DC PCF in the case of nonlinear propagation without consideration of the spectral transformations. The theory of nonlinear couplers is well summarized in [7], where the most of theoretical analysis take into account only identical cores, i.e. a symmetrical structure. In this paper we present the numerical analysis of both the temporal and the spectral nonlinear evolution of femtosecond pulses in a dual core fibre involving both dispersion and the coupling effects. Additionally, our complex experimental study demonstrates the switching of narrow spectral components shifted from the excitation wavelength by intensity or polarization changes of the input pulses. The simulation work supports the experimental results and outcomes point out on the possibility of further improvement of the dual core fibre output field control.

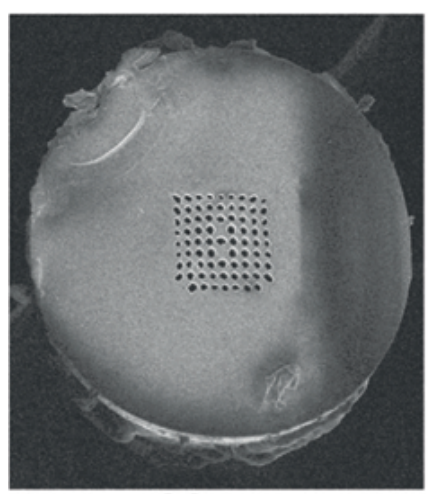

$20 \mu \mathrm{m}$

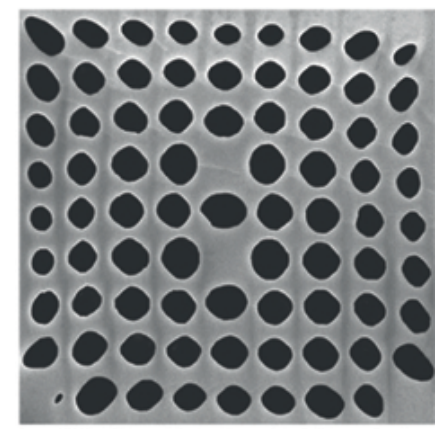

$10 \mu \mathrm{m}$
FIG. 1 SEM pictures of studied fibre and its photonic structure at two different magnifications.

\section{OBJECTIVES AND METHODS}

\subsection{Dual core fibre properties}

The nonlinear propagation study is performed on a dual core PCF structure made of $9 \times 9$ capillaries arranged in a square lattice (Figure 1). The diameter of the air holes is approximately $1.4 \mu \mathrm{m}$ with a lattice pitch of about $2 \mu \mathrm{m}$. The overall diameter of the fibre is $70 \mu \mathrm{m}$ and all experimental results presented in this paper were obtained at $5 \mathrm{~cm}$ fibre length. The cores are formed by two missing holes in the central part of the structure separated by single air hole. The fibre is made of the multi-component silicate soft glass PBG01 with composition $\mathrm{Ga}_{2} \mathrm{O}_{3}-\mathrm{PbO}-\mathrm{Bi}_{2} \mathrm{O}_{3} \mathrm{SiO}_{2}-\mathrm{Al}_{2} \mathrm{O}_{3}-\mathrm{PbO}-\mathrm{Na}_{2} \mathrm{O}-\mathrm{K}_{2} \mathrm{O}$ [10]. The Sellmeier coefficients defining the glass dispersion were determined in the visible region fulfilling the formula

$$
n(\lambda)=1+\sum_{j=1}^{3} \frac{B_{j} \lambda^{2}}{\lambda^{2}-\lambda_{j}^{2}} .
$$

The values of the Sellmeier coefficients for the PBG01 glass are $B_{1}=0.71584, B_{2}=0.70795, B_{3}=29.94204, \lambda_{1}=0.14138 \mu \mathrm{m}$, $\lambda_{2}=0.08856 \mu \mathrm{m}, \lambda_{3}=7.53 \times 10^{15} \mu \mathrm{m}$.

The fibre shown in Figure 1 was chosen for experiments for a couple of reasons. The square lattice structure was utilized due to it being more suited for the polarization properties analysis than a hexagonal structure. The overall structure dimensions were chosen for the best coupling properties from various structure sizes. Smaller structures than that presented in Figure 1 were experimentally almost uncontrollable while larger structures had worse guiding and coupling properties and did not show anomalous dispersion behaviour in the spectral range of interest [11]. The material dispersion of the fibre glass is the crucial factor in the determination of the linear properties of the fibre by numerical simulations. The linear parameters required for further simulations of nonlinear pulse propagation are: the propagation constants $(\beta)$, coupling coefficients $(\kappa)$ and effective modal areas $\left(A_{\text {eff }}\right)$ of each core. The linear properties of the fibre were calculated for each core on the basis of the material dispersion and the geometrical structure of the fibre obtained from the SEM picture (Figure 1) by means of the commercial software Mode Solutions from Lumerical. Mode Solutions provides the propagation constants and modal distributions of the supermodes. Since 
the dispersion properties of each separate core are required for the nonlinear propagation model, the dispersion properties of the separate cores were calculated by modification of the dual core structure. A circular hole was added to the opposite core to that being studied following the periodicity and dimensions of the neighbouring holes of the structure.

The theoretical approach describing the nonlinear propagation is based on the coupled generalized nonlinear Schrödinger equations (GNLSE) which are scalar allowing polarization effects to be neglected. Nevertheless there is a possibility to partially analyze the polarization effects impact by applying the dispersion properties of the two orthogonal polarization components separately. In our results, the polarization state parallel to the two core axis is assigned as $\mathrm{Y}$, while the orthogonal one is $X$. Since the dispersion properties of each core are very similar to each other, as presented in Figure 2(a), the values of the two coupling coefficients are also very close to each other. According to our calculations for $\kappa$ defined as $\kappa=\sqrt{\kappa^{(1)} \mathcal{K}^{(2)}}$ the differences of real coupling coefficients from the central function $\kappa$ are at least four orders smaller than $\kappa\left(\left|\kappa-\kappa^{(1)}\right| \approx\left|\kappa-\kappa^{(2)}\right| \leq 10^{-4} \kappa\right)$. Considering the similarity, equal coupling coefficients for both cores were used $\left(\kappa=\kappa^{(1)}=\kappa^{(2)}\right)$ utilizing a simple formula

$$
\kappa=\frac{\sqrt{\left(\beta_{S}-\beta_{A}\right)^{2}-\left(\beta^{(1)}-\beta^{(2)}\right)^{2}}}{2},
$$

which was derived from Eq. (2).

The fibre attenuation was determined experimentally in the $S$ and C-band of the near infrared spectral region as $50 \mathrm{~dB} / \mathrm{m}$, while no spectral dependence of the loss was observed. The nonlinear refractive index of the multicomponent glass was obtained from [12], where the z-scan based method resulted in a value of $n_{2}=2.5 \times 10^{-20} \mathrm{~m}^{2} / \mathrm{W}$. As the Raman response function is unknown for the PBG01 glass, and considering the similarity of both the linear and nonlinear refractive indices to those of pure silica, the Raman coefficients of silica have been used to model the Raman response function $h_{R}(t)$.

\subsection{Numerical analysis}

In order to analyze nonlinear propagation in dual core fibres, coupled GNLSEs have been derived in the form

$$
\begin{aligned}
\frac{\partial A^{(r)}}{\partial z}= & i(-1)^{r+1} \delta_{0} A^{(r)}+(-1)^{r} \delta_{1} \frac{\partial A^{(r)}}{\partial T} \\
& +\sum_{p \geq 2} \frac{i^{p+1}}{p !} \beta_{p}^{(r)} \frac{\partial^{p} A^{(r)}}{\partial T^{p}}-\frac{\alpha_{0}^{(r)}}{2} A^{(r)} \\
& +\sum_{q \geq 0} \frac{i^{q+1}}{q !} \kappa_{q}^{(r)} \frac{\partial^{q} A^{(3-r)}}{\partial T^{q}}+i \gamma^{(r)}\left(1+\frac{i}{\omega_{0}} \frac{\partial}{\partial T}\right) \\
& \times A^{(r)} \int_{-\infty}^{\infty} R\left(T^{\prime}\right)\left|A^{(r)}\left(z, T-T^{\prime}\right)\right|^{2} d T^{\prime}
\end{aligned}
$$

where $r=1,2$ and superscripts ${ }^{(1)},(2)$ indicate the fibre cores, $A$ is the amplitude of electric field, $\delta_{0}$ depends on phase velocity difference between the cores, $\delta_{1}$ depends on group velocity difference between the cores, $\alpha_{0}$ is the loss coefficient, $\kappa$ is the
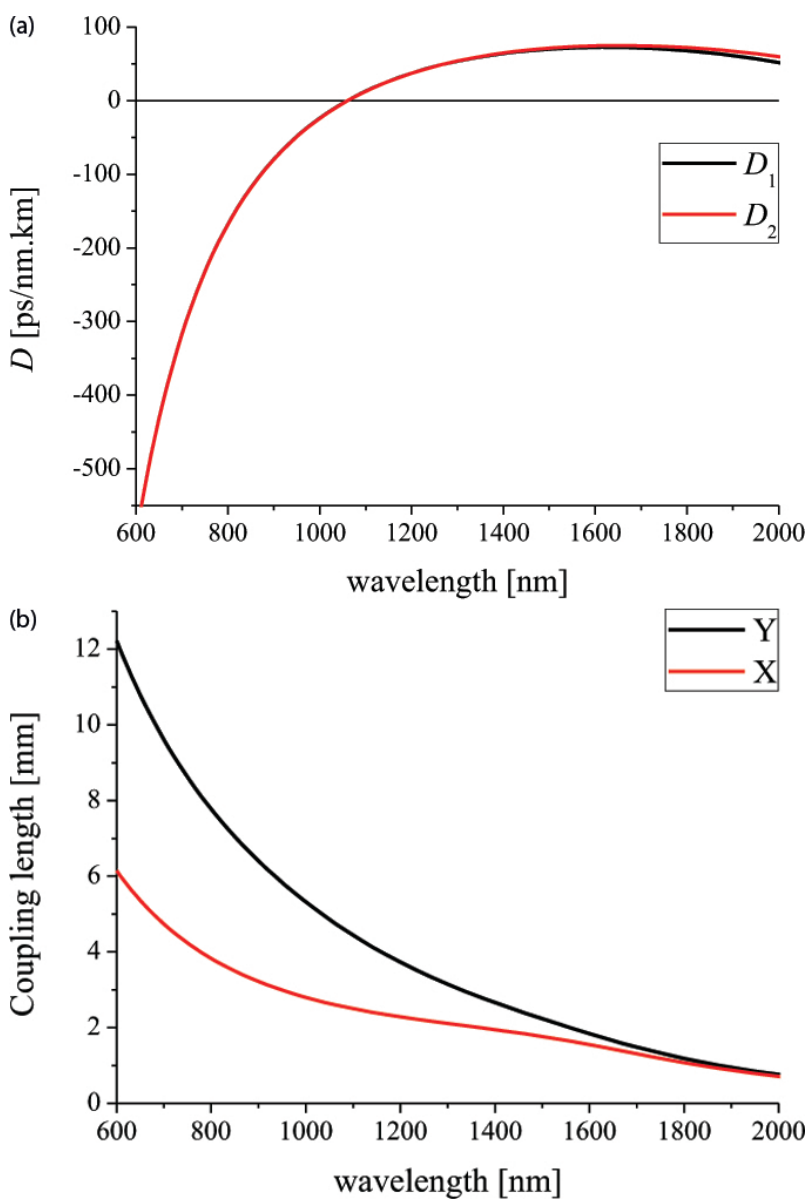

FIG. 2 Calculated dispersion characteristics (a) of each core for polarization parallel with cores - Y direction and coupling length characteristics (b) for both orthogonal polarizations.

coupling coefficient, $\gamma$ is the nonlinear coefficient and $R$ is the nonlinear response function of the material. Deeper explanation of individual terms and coefficients can be found in our previous article [13].

In order to model the fibre properties the following assumptions were utilized in our simulations. Coefficients $\delta_{0}$ and $\delta_{1}$ were included as nonzero in order to model the slight difference of optical properties of the two cores. Their nonzero values cause also the decrease of coupling efficiency between the cores [7]. Propagation constants $\beta^{(1)}$ and $\beta^{(2)}$ were included as explicit functions of frequency as well as coupling coefficient $\kappa$. They were included as results from linear simulations and interpolated to our frequency mesh in split-step method. The values of nonlinear coefficients have been determined on the base of linear simulations in combination with nonlinear refractive index value for the PBG01 glass, what resulted in values $\gamma^{(1)}=30.5 \mathrm{~W}^{-1} \mathrm{~km}^{-1}$ and $\gamma^{(2)}=30.4 \mathrm{~W}^{-1} \mathrm{~km}^{-1}$.

As the system of two Eqs. (4) cannot be solved analytically, a numerical approach based on the symmetrised split-step Fourier method modified to the matrix form [13] was utilized. The definition of the initial conditions is required in order to solve the differential Eqs. (4), i.e. the shape and duration of the input pulse needs to be defined. A hyperbolic secant pulse shape was used as the usual approximation of pulses generated by a femtosecond (fs) laser. In order to study the alloptical nonlinear coupling in dual core fibre, only one of the 


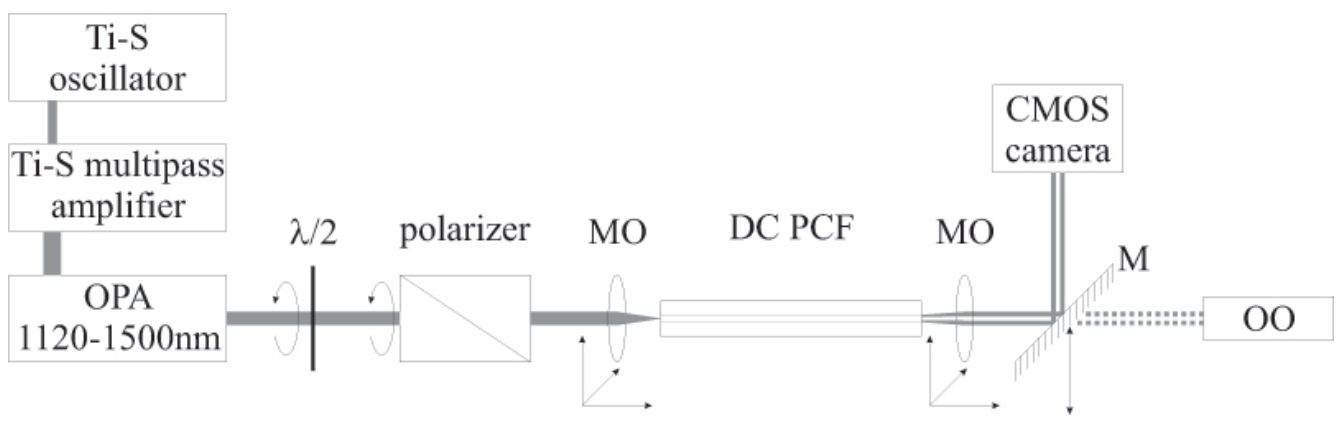

FIG. 3 Scheme of experimental setup utilized for study of nonlinear propagation of femtosecond pulses in DC PCF.

cores is excited at the fibre input. The initial conditions can be expressed by the formulae

$$
\begin{aligned}
A^{(r)}(z=0, T) & =A_{0} \operatorname{sech} \frac{T}{T_{0}} \\
A^{(3-r)}(z=0, T) & =0
\end{aligned}
$$

where $r=1,2$ and $A_{0}=\sqrt{P}$, while $P$ is the input peak power of the pulse. The pulse width $T_{F W H M}$ utilized in simulations is $80 \mathrm{fs}$ according to earlier performed experimental determination [14]. Hyperbolic secant pulses fulfil the condition $T_{F W H M}=2 \ln (1+\sqrt{2}) T_{0} \approx 1.763 T_{0}$. Input pulses have no chirp or spectral noise applied. The applied model allows determination of the spectral and temporal evolution of the input pulse along the fibre length. The input pulse and fibre properties were chosen to achieve the similar conditions to the experiments performed simultaneously with the simulations.

\subsection{Experimental study}

The experimental setup utilized for the nonlinear propagation study of the DC PCF is depicted in Figure 3. The femtosecond laser system providing the excitation pulses consists of a Ti:Sapphire oscillator, Ti:Sapphire multi-pass amplifier and BBO based optical parametric amplifier (OPA). The output pulses generated by the femtosecond laser have 80 fs FWHM with a repetition frequency of $1 \mathrm{kHz}$ in the microjoule energy range. The central wavelength of the pulses is tuneable in the range $1120 \mathrm{~nm}-1500 \mathrm{~nm}$. A half-wave retarder $(\lambda / 2)$ and Glan polarizer at the output of the laser system were used in order to change the polarization and intensity of the laser light. A micro-objective (MO) with a magnification of $40 \times$ was employed to couple the light into the fibre and selectively excite one of the two cores. The second $40 \times$ micro-objective was placed at the output to project the fibre end near-field distribution on the collection fibre of the Ocean Optics IR spectral analyzer (OO). Optionally, a mirror was inserted into the output beam to direct the generated light towards a CMOS camera. The camera recorded the spatial light distribution mainly in the VIS and near IR as it contains a Si-based CMOS chip. The collection fibre input and CMOS chip were at the same distance from the mirror to assure they are both placed in the image plane of the output micro-objective. The projected near field image of the DC PCF output was magnified to the millimetre scale enabling selective spectral registration from each of the two fibre cores.

During the measurements the OPA was tuned to $1300 \mathrm{~nm}$ cen- tral wavelength and the pulse energies reached the few hundred $n J$ region before the input micro-objective. The excitation pulses exhibited quite large intensity fluctuation at the level of $\pm 20 \%$, which has a significant effect to the output nonlinearly broadened spectrum registered mostly with $10 \mathrm{~s}$ average time [13]. The absolute peak intensity at the input of the fibre is unknown as the coupling efficiency into the fibre is unknown. Therefore only relative changes of input intensity were monitored. The experimental study included different input settings such as polarization, intensity, selective input coupling into each of the fibre cores and selective detection of spectra from each core. The analysis of the registered spectra is focused on comparison of spectra between the two output channels with emphasize on the switching performance by changing the input parameters.

\section{RESULTS AND DISCUSSION}

A complex experimental study of the dual core nonlinear propagation was performed focusing on the effect of input intensity and polarization direction changes at selective excitation of the two input fibre channels. An example of input intensity dependent spectral changes for input polarization parallel to the fibre cores ( $Y$ polarization) registered separately for the both output channels is presented in Figure 4. The three letters in the title of each graph define in consecutive order the polarization of the input light ( $\mathrm{Y}$ or $\mathrm{X})$, the excited input core ( $\mathrm{U}$ - upper or $\mathrm{L}$ - lower) and the registered output core ( $\mathrm{U}$ or $\mathrm{L})$. The intensity values in Figure 4 are relative, as there was no possibility to measure the absolute intensity coupled into the fibre in our experimental setup. The intensity of the light was measured before the fibre and the intensity change before the fibre is assumed to be proportional to the intensity change coupled into the fibre upon unchanged fibre excitation geometry. The results are normalized to the peak intensity of all the spectra in Figure 4; Figures 6, 7, 8 and 9 are normalized in the same manner. The noise recorded at low intensities is produced by the spectral analyzer, as the measured intensities were low in comparison to its sensitivity. Therefore $10 \mathrm{~s}$ integration time intervals (10000 pulses in one measurement) were required. Significant spectral broadening with increasing input intensity is obvious, which is the standard phenomenon observed in the nonlinear propagation regime. There is a possibility to distinguish separate spectral features, whose quantity rises with increasing intensity. The broadening process is quite symmetric around the central wavelength $1300 \mathrm{~nm}$, which suggests the high influence 
(a)
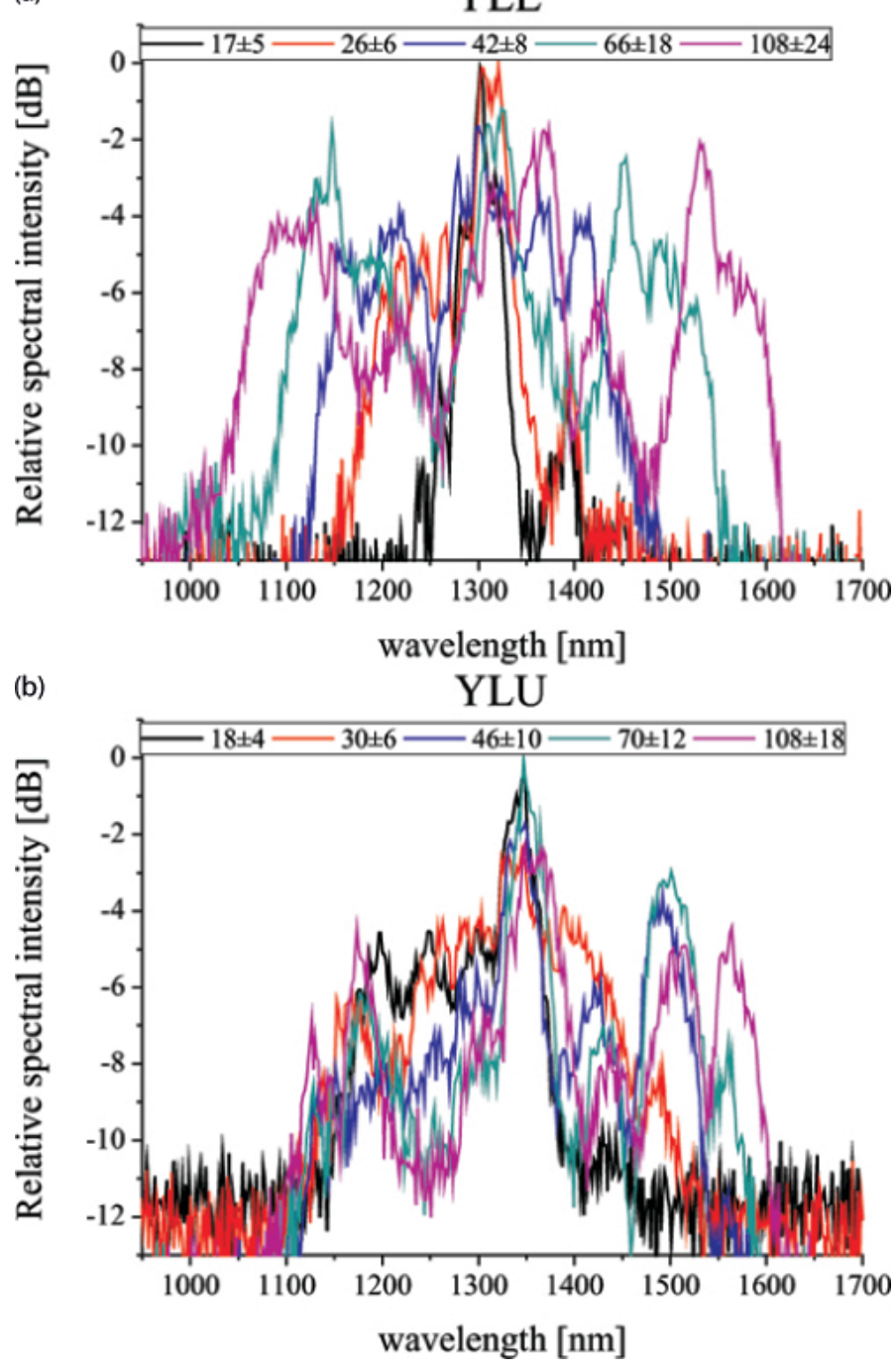

FIG. 4 Experimentally recorded spectra for $Y$ polarization at the output of the fibre with excited lower core - L. (a) recorded spectra in excited lower core - L, (b) recorded spectra in non-excited upper core - $U$, for different input peak intensities.

of the GVD term on broadening through the self-phase modulation (SPM) effect. Considering the zero dispersion point at around $1060 \mathrm{~nm}$ wavelength, which was obtained from the theoretical model, GVD at central wavelength $1300 \mathrm{~nm}$ is quite high in both cores reaching values about $-48 \mathrm{ps}^{2} / \mathrm{km}$ $(\mathrm{D}=54 \mathrm{ps} /(\mathrm{nm} . \mathrm{km}))$, therefore the GVD term is the most significant dispersion term. The third order dispersion term causing asymmetric broadening has a smaller impact on the resulting spectrum. Higher order dispersion terms influence mainly pulses spanning over one octave i.e. in our case mainly the wavelength of the generated dispersion wave which lies beyond the scope of this paper. Propagation in the anomalous dispersion region suggests solitary wave generation and fission processes that together with Raman self-frequency shift cause the occurrence of separated spectral features with their slight red shift along the fibre. The dispersion wave generated during the solitary wave fission process is not observable on the measured spectra as the lower limit of the spectrometer sensitivity is at $900 \mathrm{~nm}$.

The numerical simulations of the nonlinear propagation were based on the simulation outputs of the fibre linear properties (Figure 2). The results from the nonlinear simulation propaga- tion correspond to the above explanation of the nonlinear interaction. Figure 5 shows the 3D maps describing the dual core propagation along the fibre both in the temporal and spectral domains at similar excitation conditions to the experimental results (1300 nm, Y polarization). The imaging of the fibre surface at the output did not allow the identification of the fibre cores during the experiments. Therefore it was not possible to determine which fibre core was excited in correspondence to the presented SEM picture on Figure 1. In the simulation results are the fibre cores therefore labelled as $1^{\text {st }}$ and $2^{\text {nd }}$, without clear correspondence to the experimentally examined upper and lower core.

The input intensity in presented simulations was chosen in order to reach the same level of spectral broadening as observed experimentally in the NIR region, which resulted in a $24 \mathrm{~kW}$ input peak power coupled to the fibre. According to Figure 5, two regions of propagation are distinguishable. At the beginning of the pulse propagation there is observable rapid high order solitary wave generation and spectral broadening. At about $1 \mathrm{~cm}$ of fibre length, generation of a dispersion wave and solitary wave fission process takes place. After solitary wave fission, the individual spectral features propagate almost independently of each other. A slight Raman selffrequency shift is observable in this area of propagation on the spectral maps of Figure 5 with vanishing character due to the significant fibre attenuation. Raman self frequency shift can be observed on the temporal maps mainly on the most significant solitary wave. Due to the speed change of that solitary wave caused by the spectral shift, its slope is decreasing along the propagation represented on the temporal map. Features with even lower speed on the right side of each temporal map are dispersive waves generated during the solitary wave fission. Dispersive waves have a constant slope on the temporal map, thus they propagate with constant speed and are not affected by further spectral changes and nonlinear effects. Solitary oscillation periods are not observable, mainly because they are overlaid by the more pronounced effect of coupling oscillations between the cores. The spectral transformations after $1 \mathrm{~cm}$ of the fibre are affected mainly by the dual core propagation. Based on the simulations of the linear properties of the fibre in Figure 2(b), longer coupling lengths are expected for shorter wavelength ranges. The obtained temporal and spectral maps of Figure 5 are in correspondence with the presented concept, as the coupling oscillation period monotonically increases to shorter wavelengths. The most intensive spectral feature on the red side of spectra at about $1500 \mathrm{~nm}$ expresses coupling length values of about $2 \mathrm{~mm}$, while dispersion wave at about $700 \mathrm{~nm}$ expresses coupling length of about $10 \mathrm{~mm}$.

According to the spectral maps of Figure 5 it is obvious that the dual core nonlinear propagation in the anomalous region supports significant spectral broadening. Additionally in the case of dual core fibres the spectral broadening is influenced by wavelength dependent coupling oscillations of the generated spectral features. Based on this concept, the different spectral features exhibit different ratio of spectral intensity in the two output channels depending on the relation between the wavelength specific coupling and the fibre length. The complexity of the described pulse propaga- 
(a)

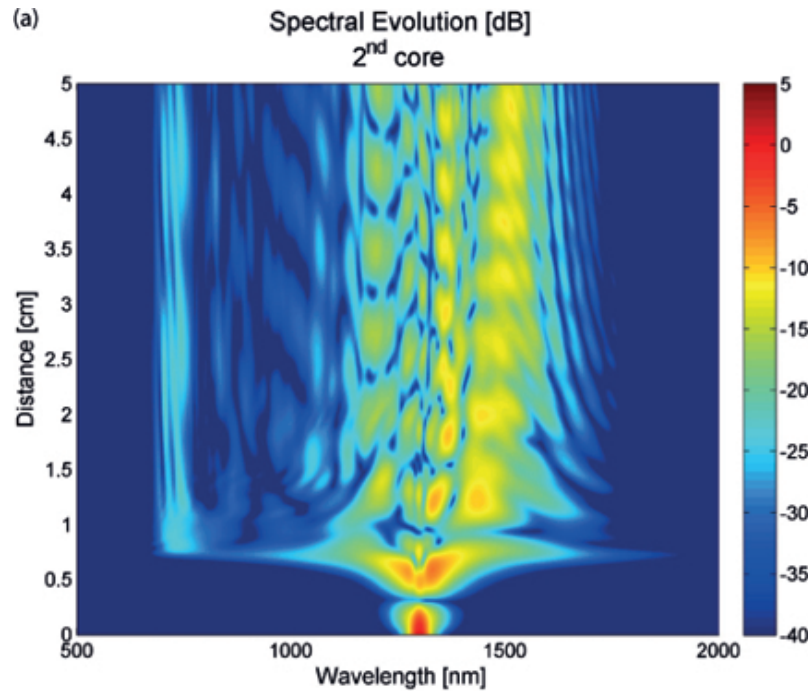

(c)

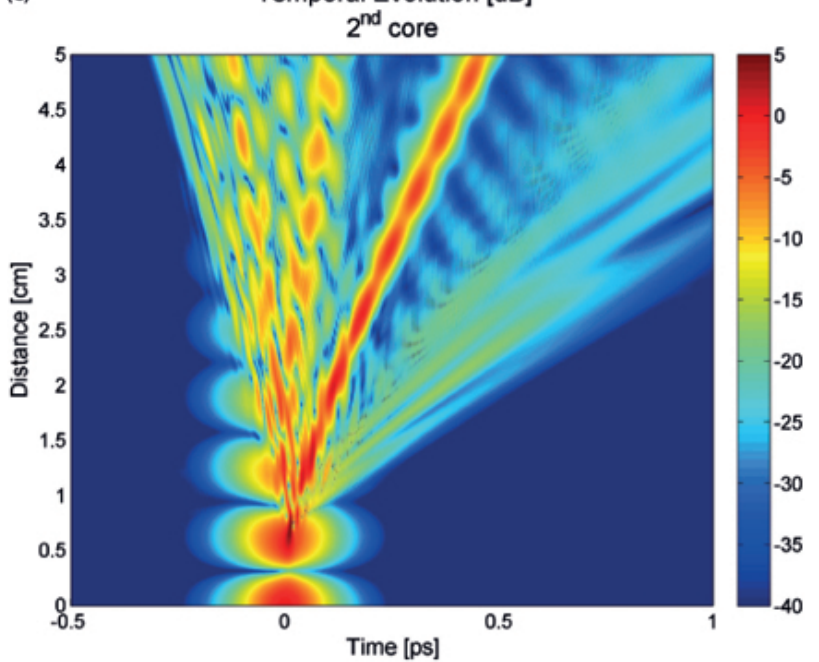

(b)

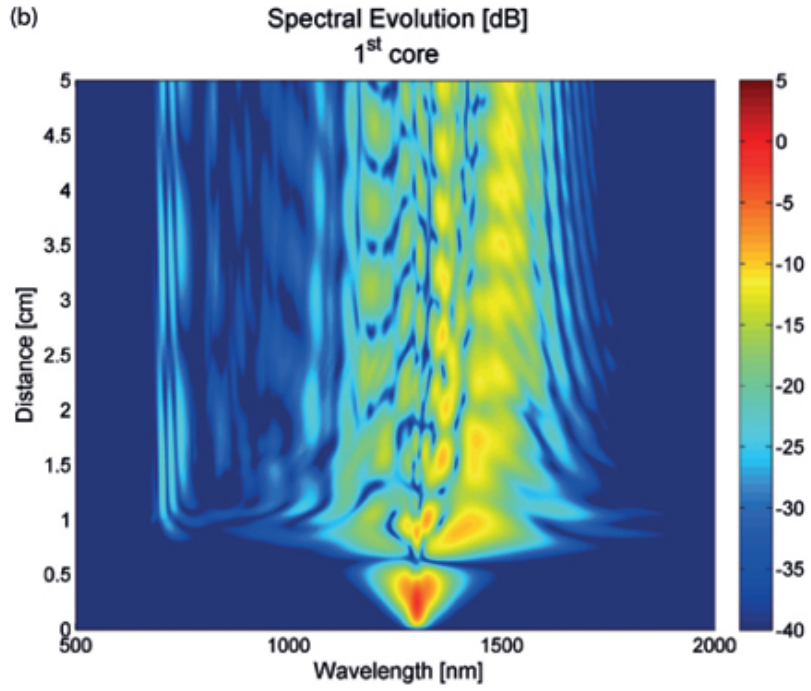

(d)

Temporal Evolution [dB] $1^{\text {st }}$ core

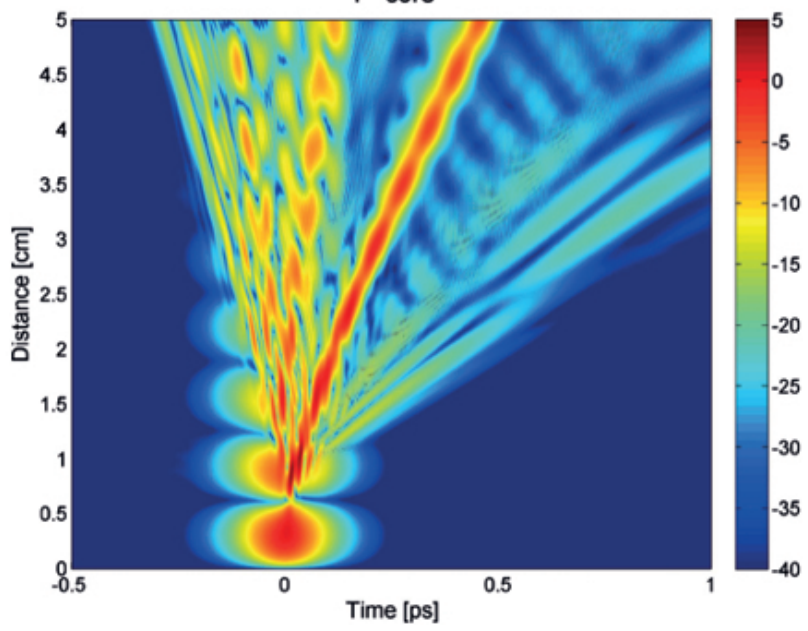

FIC. 5 Spectral and temporal maps of nonlinear pulse propagation in each core of the DC PCF obtained from numerical simulations for 24 kW input peak power coupled to the fibre, $1^{\text {st }}$ core is the upper one in the Figure 1 without clear correspondence to upper and lower cores from the experiment.

tion scenario promises the possibility of the controlled spectral intensity switch of femtosecond pulses shifted from the excitation wavelength. Analysis of the spectrograms obtained both experimentally and theoretically was performed, in order to identify the possibilities for the envisaged all-optical field switching between the two fibre cores.

Figure 6 presents the potential of the special DC PCF for the nonlinear switching application at short sample lengths focusing only on the excitation spectral area. Comparison of the spectral intensities in the two fibre cores is shown at $12 \mathrm{~kW}$ and $21 \mathrm{~kW}$ input peak powers at a distance of $11.4 \mathrm{~mm}$ from the fibre input. According to the spectral intensity graphs it is obvious that the spectral intensity is similar in both fibre cores in the vicinity of the pump wavelength at low peak power. On the other hand, it is possible to considerably reduce the spectral intensity around $1290 \mathrm{~nm}$ in the nonexcited fibre core by increasing the input peak power and to attain an intensity ratio between the cores of greater than $10 \mathrm{~dB}$. These findings point to the fact, that the employed DC PCF structure is close to the optimum regarding the nonlinear coupler performance at the studied excitation wavelength using a fibre length corresponding to a few coupling lengths. However, the numerical prediction presented in Figure 6 does not represent large progress in relation to earlier nonlinear coupler achievements. The aim of this work was to extend the nonlinear coupling performance for spectral regions shifted significantly from the pump wavelength. From the simulation results in Figure 6 there is no signature of the spectral switching performance at any shifted wavelength because the short fibre length does not ensure sufficient change in the pulse spectrum. Our systematic numerical study revealed that elongation of the double core propagation length results in solitary wave self-frequency shift approaching the wavelength of $1500 \mathrm{~nm}$ (Figure 5). A propagation distance of $5 \mathrm{~cm}$ ensures a sufficient space for nonlinear coupling performance based on the coupling length dispersion. An input peak power increase results in a slight wavelength shift of the dominant solitary wave, which allows the realization of a spectral switch in the vicinity of the S-band of optical communication systems.

Following this aim in Figure 7(a) two experimental spectra are compared. The spectra were obtained by separate registration at the output of two fibre cores, with the same excitation, same polarization direction and almost the same peak intensity for a $5 \mathrm{~cm}$ fibre length. The comparison of spectra indicates strong 

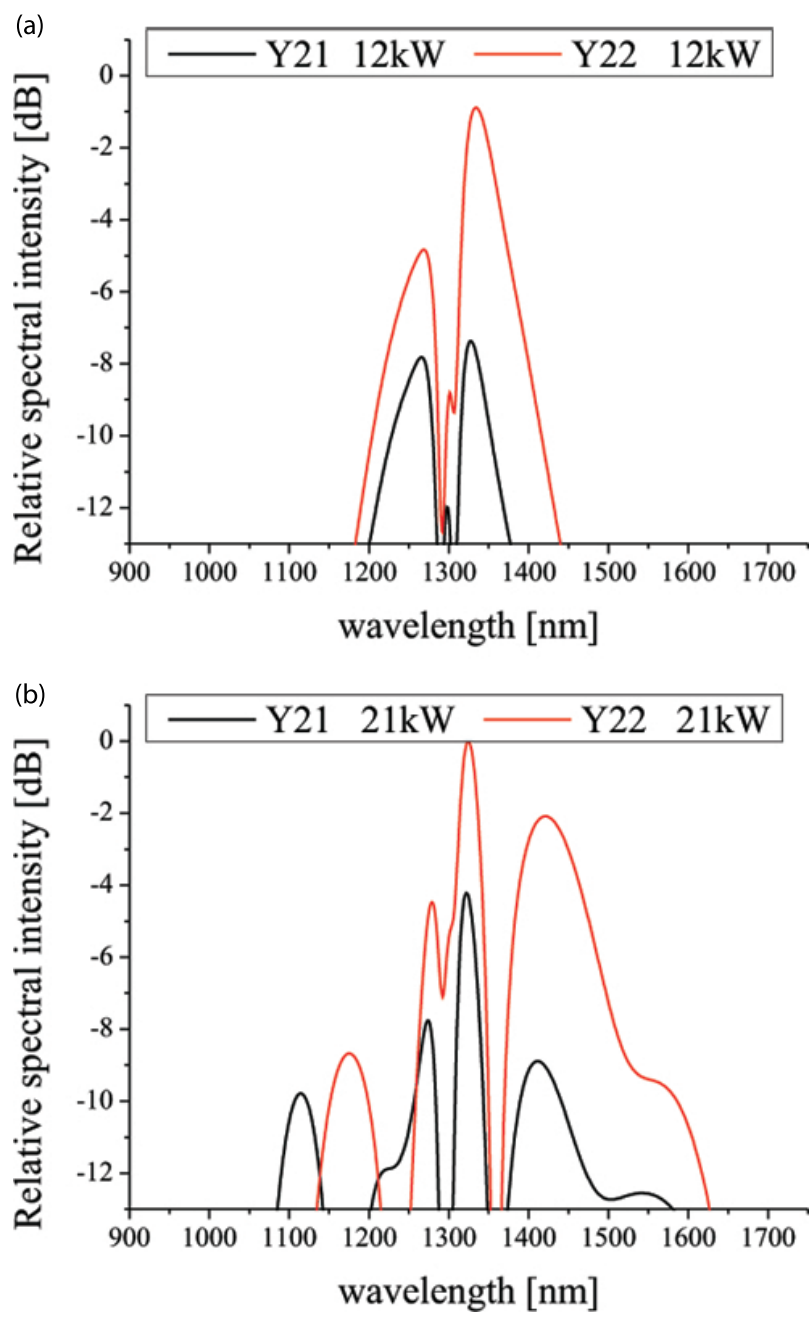

FIG. 6 Simulation spectra exhibiting nonlinear coupler performance at $1290 \mathrm{~nm}$ wavelength for $Y$ polarization with excited $2^{\text {nd }}$ core after $11.4 \mathrm{~mm}$ propagation distance. In the lower intensity case (a) the output intensity ratio is only about $1 \mathrm{~dB}$ and in the higher intensity case (b) the spectral intensity in the excited $2^{\text {nd }}$ core is more than 10 $\mathrm{dB}$ higher than in non-excited $1^{\text {st }}$ core.

spectral features in the non-excited core at $1500 \mathrm{~nm}$ wavelength, which is absent in the excited core. The intensity ratio at $1500 \mathrm{~nm}$ between the two cores is about $10 \mathrm{~dB}$. Increasing the input intensity by approximately $50 \%$ changes the intensity in the excited core - reaching approximately the same level as in the non-excited core with an intensity ratio between the cores lower than $2 \mathrm{~dB}$ (Figure 7(b)). The results are in correspondence with our knowledge about the dual core nonlinear propagation. The nonlinear interaction allows the spectral intensity redistribution between the cores for different reasons. The increase of input power changes the position, where the solitary wave fission effect occurs along the fibre, determining thus the further coupling scenario. Further increase of the input power may cause higher intensities than the critical power level [7], however in the case of the all presented results we worked with input peak intensities firmly below this level estimating its value to be around $75 \mathrm{~kW}$. Below the critical power, the intensity induced change of the position of solitary wave fission varies the final intensity distribution between the cores. Additionally, the increase of the input power modifies the coupling length spectral characteristics by inducing a nonlinear change of the refractive index which also varies the spectral feature redistribution between the cores at the fi-
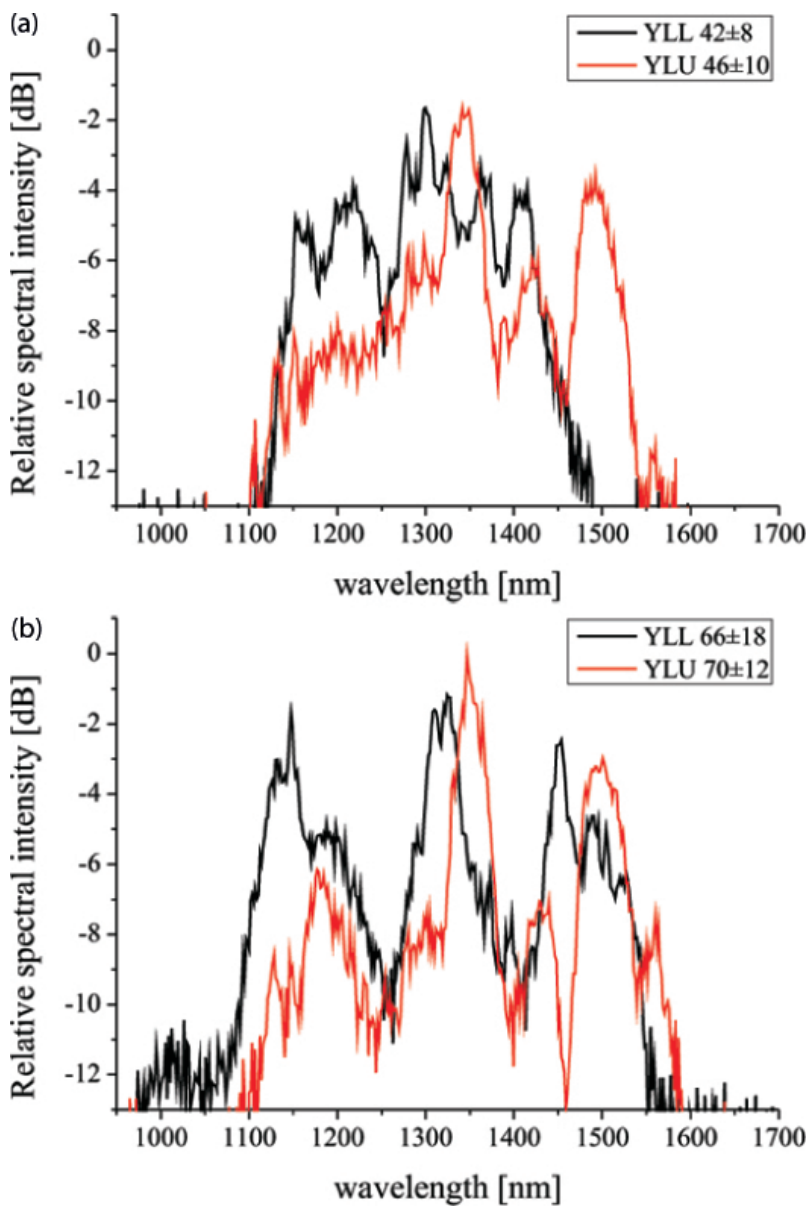

FIG. 7 Experimentally obtained spectra proposing all-optical intensity switch between the two fibre cores at $1500 \mathrm{~nm}$ wavelength for Y polarization and lower core excited - L. The numbers in legend determine relative intensity. In the lower intensity case (a) the spectral intensity in the excited lower core $L$ is about $10 \mathrm{~dB}$ less than in non-excited upper core $\mathrm{U}$. In the higher intensity case (b) the intensity ratio between the cores is only about $2 \mathrm{~dB}$.

bre end. Both of these mechanisms represent a potential for a spectral intensity switch beyond the excitation spectral area which has been confirmed by experiment. According to the obtained results applying the proper input polarization and intensities to the dual core PCF together with a narrow band filter allows the creation of a high contrast all optical switch controlled by intensity.

The intensity controlled switching can be demonstrated also by simulation without an exact match to the experimental conditions as there are some uncertain parameters. Beside the lack of information about the absolute input intensity, further uncertainty originates from the coupling length determination. For example, the coupling length for $\mathrm{Y}$ polarization is about $3 \mathrm{~mm}$ at $1500 \mathrm{~nm}$ wavelength (Figure 1). Considering a $5 \mathrm{~cm}$ fibre length with solitary wave fission, which occurs after $1 \mathrm{~cm}$ propagation and generates a $1500 \mathrm{~nm}$ spectral feature, there are $4 \mathrm{~cm}$ remaining distance for intensity oscillations between the cores, what is roughly 13 coupling lengths - about 7 oscillations (Figure 5). According to the described nonlinear propagation mechanism, we neglect the nonlinear contribution to the coupling length after solitary wave fission as the intensity of the $1500 \mathrm{~nm}$ feature is low. Taking into account only the linear coupling behaviour together with the 

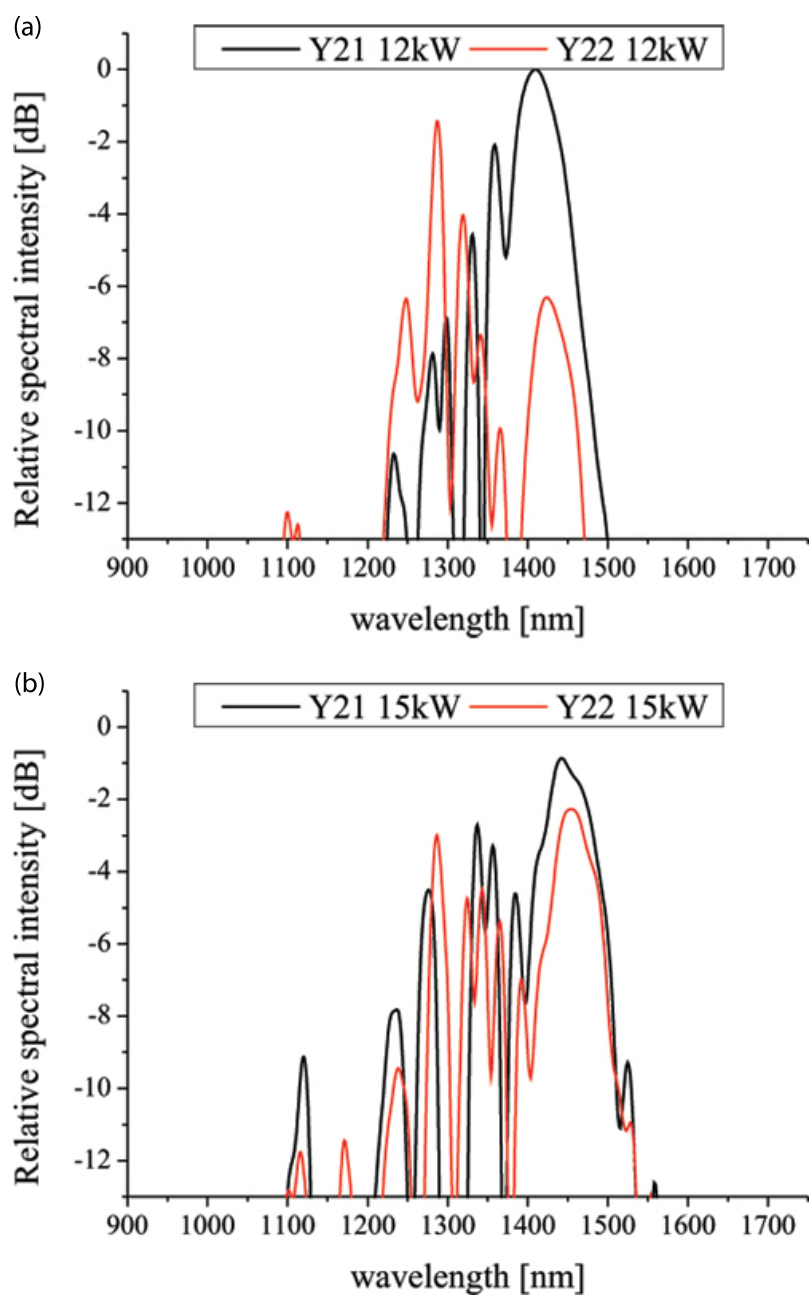

FIG. 8 Simulation spectra proposing all-optical intensity switch between the two fibre cores at $1390 \mathrm{~nm}$ wavelength for $Y$ polarization with excited $2^{\text {nd }}$ core. In the lower intensity case (a) the spectral intensity in the excited $2^{\text {nd }}$ core is about $10 \mathrm{~dB}$ less than in non-excited $1^{\text {st }}$ core. In the higher intensity case (b) the output intensity ratio is only about $1 \mathrm{~dB}$.

uncertainty in the coupling length (at a level of 3.5\%) after 7 periods of coupling oscillation, the information about the position of $1500 \mathrm{~nm}$ spectral component between the cores is completely unknown. Considering the very strong dependence of intensity oscillations on coupling length characteristics, the uncertainty does not allow the reliable comparison of the experimental and simulation results. Additionally, the experimentally obtained output spectra are smoother than the simulated ones due to the input intensity fluctuations during the experiment and their averaging in the measurement results, which was analyzed in our previous article [13]. Due to these two main reasons, there is no point in trying to exactly compare simulation and experimental spectra that are quantitatively very different due to the mentioned uncertainties. On the other hand, the simulations can help to explain and understand the role of different processes inside the fibre and help to analyze the stability of results against uncertainty of individual parameters.

The simulation spectra in Figure 8 suggest the possibility of manufacturing a high contrast intensity switch at $1390 \mathrm{~nm}$ for the same fibre length as in the experiment. At $12 \mathrm{~kW}$ input peak intensity the output spectral intensities ratio between the
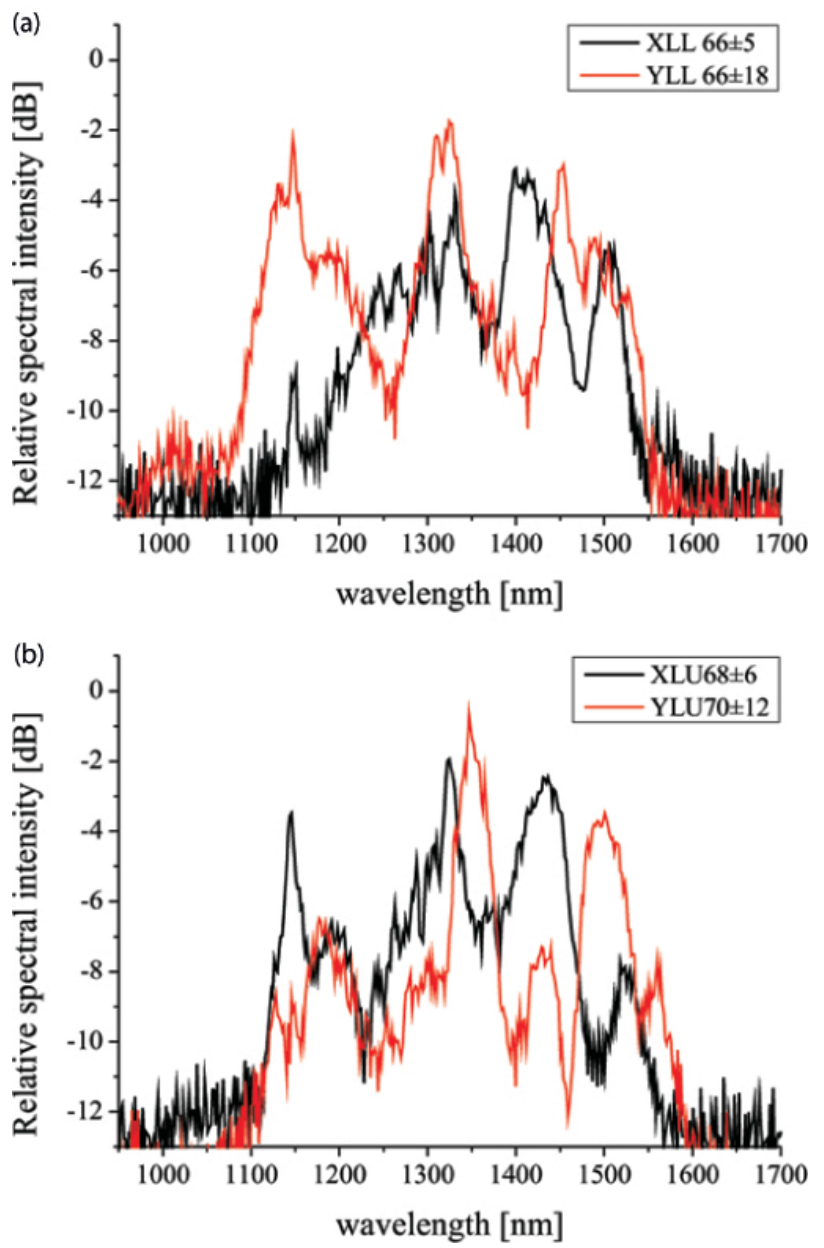

FIG. 9 Experimentally obtained spectra proposing all-optical polarization switch between the two fibre cores at $1500 \mathrm{~nm}$ wavelength. In the excited and monitored lower core $L(a)$ input polarization direction does not change the output spectral intensity. In the non-excited upper core $U$ (b) the intensity ratio is about $7 \mathrm{~dB}$.

cores is about $10 \mathrm{~dB}$, while at $15 \mathrm{~kW}$ the ratio is about $1 \mathrm{~dB}$. Figure 8 presents the theoretical confirmation of the application potential for significant change of spectral intensity ratio by alternating the input intensity. According to the coupling theory [7], optimization of the fibre length for a given fibre leads to the possibility of production a narrow band all-optical switch at arbitrary frequency in the nonlinearly broadened spectral range. The polarization switch is quite evident in simulations taking into account the different coupling length characteristics of the two orthogonal polarization directions presented on Figure 2(b). In this case the experimental registration of the effect is more valuable, what confirms the application potential of polarization switching approach.

Polarization affected narrow band all-optical switching between the cores is based on the rotation of the input pulse polarization direction. The concept is supported by the significant difference between the coupling lengths of the two main polarization axes of the fibre (Figure 2(b)). The possibility for continuous wavelength tuning of the visible dispersion wave by rotation of the input polarization direction was shown in our earlier work [11]. However, in this paper our focus is the investigation of the possibility for all-optical switching in the NIR spectral region by changing the polarization direction between two orthogonal states. Figure 9 shows the 
experimentally obtained spectral intensity curves comparing two perpendicular polarization states in the excited and nonexcited cores registered at the same input intensities. Focusing on the narrow-band region around $1500 \mathrm{~nm}$, in the excited core the intensity at the output is very similar in both cases - input polarization direction does not affect output intensity (Figure 9(a)). On the other hand, in the non-excited core there is about a $7 \mathrm{~dB}$ change in spectral intensity at $1500 \mathrm{~nm}$ (Figure 9(b)). In the case of a polarization switch there is also the possibility to reach the high contrast switch for arbitrary wavelength by proper setting of the fibre length and input intensity.

Figures 7, 8 and 9 show the potential for an all-optical intensity and polarization controlled narrow-band switch based on a DC PCF. As the nonlinear effects produce broadband pulses, which are influenced by the spectral dependence of the coupling and dispersion, the switch can be utilized only in a narrow spectral region. A further important aspect is that our experimental results were affected by the relatively low stability of the input laser intensity. Fluctuations of intensity caused smoother measured spectra than the ones predicted by simulations. Therefore, if the stability can be increased, the individual spectral features would be better separated and would bring better contrast to the switching performance. Despite these drawbacks, the results clearly confirm the application potential of nonlinearly controlled DC PCFs for all-optical switching. In order to manufacture the component for a specific switching task, the fibre parameters must fit the application requirements. Thanks to the many linear parameters (dispersion of each core, spectral and polarization dependence of coupling coefficients) there is a wide space for the tailoring work. Small deviations in the fabricated fibre parameters (dispersion and coupling) can be compensated for by the fibre length and input intensity. Moreover, according to our results even shorter fibre lengths are sufficient reducing the fibre losses, which will enhance the effect of the Raman based self-frequency shift. That fact further increases the application potential of our results for all-optical switching. Finally, with the combination of the presented approach and the strongly developing multi-component glass fibres with significantly higher nonlinear refraction indices, even sub-nanojoule level performance is realistic for future applications.

In summary, a complex study of nonlinear propagation of femtosecond pulses in DC PCF was realized with emphasis on application as all-optical switch. The work involved both experimental and theoretical approaches investigating the spectral and spatial evolution of $80 \mathrm{fs}$ NIR pulses along a $5 \mathrm{~cm}$ long DC PCF. The most important contribution of the experimental work is the separate excitation and spectral registration of the two fibre cores, allowing the proper comparison of the experimental and the simulation approaches. According to the obtained results the influence of the linear (coupling length characteristics, dispersion) and nonlinear (self-phase modulation, solitary wave fission, dispersion wave generation) effects on the output spectra were identified. A detailed experimental study focused on the influence of the input parameters, such as peak intensity and polarization direction, brought promising results showing the switching performance of spe- cific spectral features between the two output channels. The intensity and the polarization switch resulted in change of the spectral intensity ratio between the two cores at the level of $10 \mathrm{~dB}$ and $7 \mathrm{~dB}$ respectively, in the S-band of optical communication systems. The simulation work confirmed the potential of DC PCFs for the switching performance, which could be optimized for arbitrary spectral component of the nonlinearly broadened output spectra. Finally, the perspectives for ensuring even higher switching contrast and preparation of switching components with better parameters were presented.

\section{ACKNOWLEDGEMENTS}

The presented work was supported by research projects VEGA 1/1187/12 and 1/0907/13 (Slovak Scientific Grant Agency), MK thank to John M. Dudley for precious advices with simulations. RB was supported by research project NN515523738 (Polish Ministry of Science and Higher Education) and by the project operated within the Foundation for Polish Science Team Programme co-financed by the European Regional Development Fund, Operational Program Innovative Economy 2007-2013.

\section{References}

[1] J. C. Knight, T. A. Birks, P. St. J. Russell, and D. M. Atkin, "Allsilica single-mode optical fiber with photonic crystal cladding," Opt. Lett. 21, 1547-1549 (1996).

[2] J. C. Knight, and P. St. J. Russell, "New Ways to Guide Light," Science 296, 276-277 (2002).

[3] D. Mogilevtsev, T. A. Birks, and P. St. J. Russell, “Group-velocity dispersion in photonic crystal fibers," Opt. Lett. 23, 1662-1664 (1998).

[4] J. K. Ranka, R. S. Windeler, and A. J. Stentz, "Optical properties of high-delta air silica microstructure optical fibers," Opt. Lett. 25 , 796-798 (2000).

[5] A. Betlej, S. Suntsov, K. G. Makris, L. Jankovic, D. N. Christodoulides, G. I. Stegeman, J. Fini, et al., "Alloptical switching and multifrequency generation in a dual-core photonic crystal fibre," Opt. Lett. 31, 1480-1482 (2006).

[6] J. M. Dudley, G. Genty, and S. Coen, "Supercontinuum generation in photonic crystal fibre," Reviews of Modern Physics 78, 1135-1184 (2006).

[7] G. P. Aggrawal, Applications of Nonlinear Fibre Optics (Academic Press, New York, 2001).

[8] J. R. Salgueiro, and Y. S. Kivshar, "Nonlinear dual-core photonic crystal fiber couplers," Opt. Lett. 30, 1858-1860 (2005).

[9] M. Liu, Y. Chen, and D. Wang, “Effects of intrapulse stimulated Raman scattering and intermodal dispersion on short pulse propagation in a nonlinear two-core PCF coupler," JETP Letters 91, 548-550 (2010).

[10] R. Buczynski, "Photonic Crystal Fibres," Acta Physica Polonica A 106, 141-168 (2004).

[11] I. Bugar, D. Lorenc, I. V. Fedotov, A. B. Fedotov, R. Buczynski, D. Pysz, F. Uherek, and A. M. Zheltikov "Nonlinear frequency conversion in double core photonic crystal fibers," Proc. SPIE 6582, 16 (2007) 
[12] D. Lorenc, M. Aranyosiova, R. Buczynski, R. Stepien, I. Bugar, A. Vincze, and D. Velic, "Nonlinear refractive index of multicomponent glasses designed for fabrication of photonic crystal fibers," Appl. Phys. B 93, 531-538 (2008).

[13] M. Koys, I. Bugar, V. Mesaros, and F. Uherek "Supercontinuum generation in dual core photonic crystal fiber," Proc. SPIE 7746, 11 (2010).
[14] A. B. Fedotov, A. N. Naumov, A. M. Zheltikov, I. Bugar, D. Chorvat Jr., D. Chorvat, A. P. Tarasevitch, and D. von der Linde, "Frequency-tunable supercontinuum generation in photoniccrystal fibers by femtosecond pulses of an optical parametric amplifier," J. Opt. Soc. Am. B 19, 2156-2164 (2002). 